$10 / 8959510$
SANDIA REPORT

SAND95-8488 • UC-405

Unlimited Release

Printed July 1995

\title{
Metastable States and Intermittent Switching of Small Populations of Confined Point Vortices
}

Robert W. Schmieder

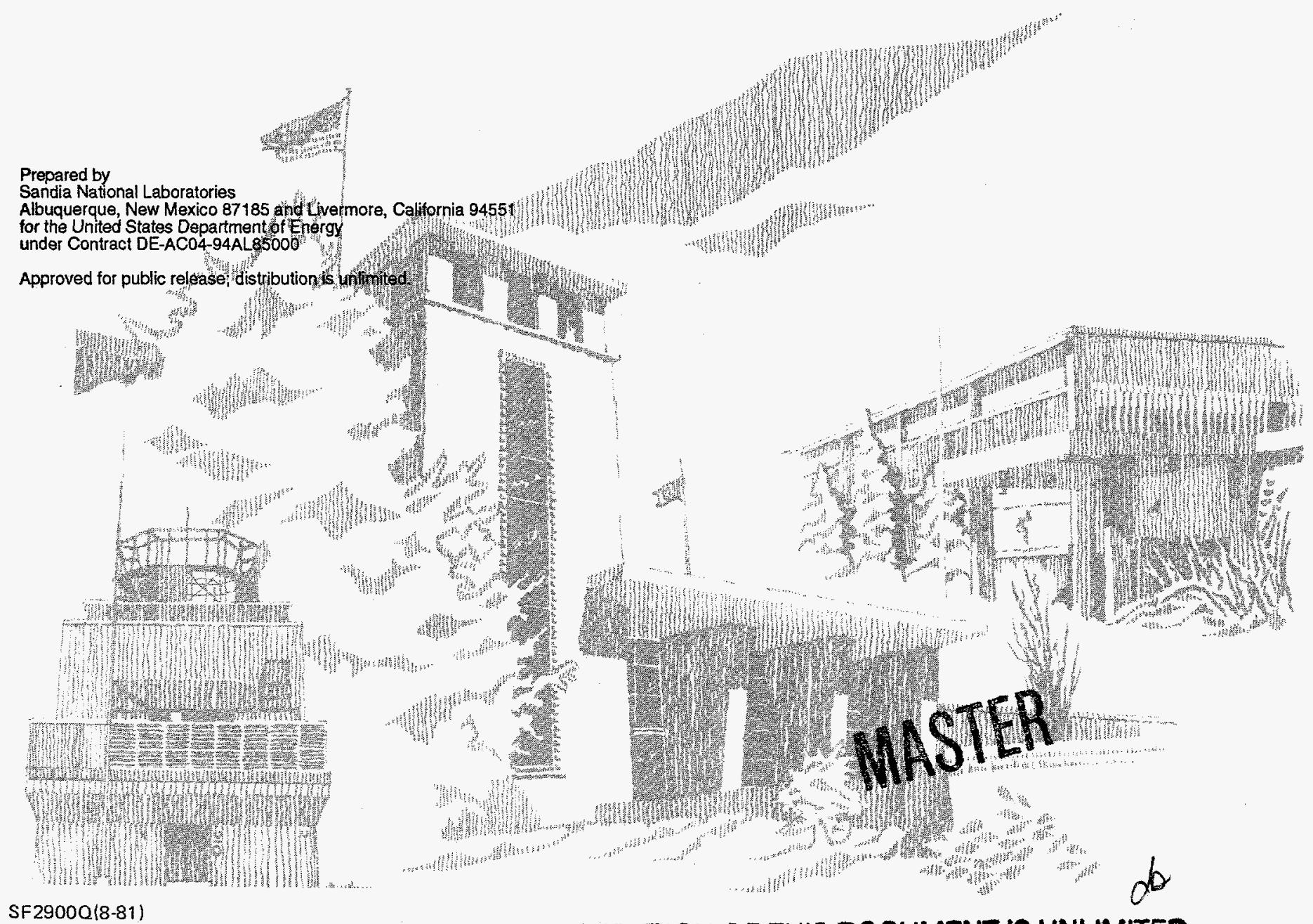




\begin{abstract}
Issued by Sandia National Laboratories, operated for the United States; Department of Energy by Sandia Corporation.

NOTICE: This report was prepared as an account of work sponsored by an agency of the United States Government. Neither the United States Government nor any agency thereof, nor any of their employees, nor any of the contractors, subcontractors, or their employees, makes any war. ranty, express or implied, or assumes any legal liability or responsibility for the accuracy, completeness, or usefulness of any information, apparatus, product, or process disclosed, or represents that its use would not infringe privately owned rights. Reference herein to any specific commercial product, process, or service by trade name, trademark, manufacturer, or otherwise, does not necessarily constitute or imply its endorsement, recommendation, or favoring by the United States Government, any agency thereof or any of their contractors or subconractors. The views and opinions expressed herein do not necessarily state or reflect those of the United States Government, any agency thereof or any of their contractors or subcontractors.
\end{abstract}

This report has been reproduced from the best available copy.

Available to DOE and DOE contractors from:

Office of Scientific and Technical Information

P. O. Box 62

Oak Ridge, TN 37831

Prices available from (615) 576-8401, FTS 626-8401

Available to the public from:

National Technical Information Service

U.S. Department of Commerce

5285 Port Royal Rd.

Springfield, VA 22161 


\section{DISCLAIMER}

Portions of this document may be illegible in electronic image products. Images are produced from the best available original document. 
SAND95-8488

UC-405

Unlimited Release

Printed July 1995

\title{
METASTABLE STATES AND INTERMITTENT SWITCHING OF SMALL POPULATIONS OF CONFINED POINT VORTICES
}

\author{
Robert W. Schmieder \\ Sandia National Laboratories \\ Livermore, CA 94551 USA \\ rwschmi@ca.sandia.gov
}

\begin{abstract}
We have found that small populations of point vortices confined in a box exhibit a variety of new and interesting metastable collective motions, ranging from rigid body rotation to complete chaos. These motions are induced by simulated heating and cooling of the vortices; they do not appear in adiabatic systems. By judicious choice of vortex circulations, heating and cooling rates, and box size, we have produced systems that switch intermittently between several metastable states, that oscillate quasi-periodically, and that show a variety of interesting collective behaviors that in some cases are suggestive of biological organisms.
\end{abstract}




\section{INTRODUCTION}

Vortices are encountered in many fields, including fluid dynamics [McWilliams \& Weiss, 1994]; Sutyrin, et al., 1994], plasmas [Glanz, 1994], superfluidity and super-conductivity [Harada, et al., 1992], and optics [Arecchi, et al., 1991; Jeffery \& D'Angelo, 1994]. Single finite vortices, often with complex internal structure, occur in planetary atmospheres [Sutyrin, 1994]; Busse, 1994], and rotating fluids [Sutyrin, \& Yushina, 1986; van Heijst, \& Kloosterziel, 1989; Hopfinger \& van Heijst, 1993]. A valuable model is that of point vortices in which multiple points produce mutually transverse forces, generally resulting in circulation around their common center [Kunin, et al., 1992]. There is a considerable literature on the motions of small systems of point vortices [Aref, 1979; Aref \& Pomphrey, 1982].

A new and difficult complication is added if the vortices are confined [Pointin \& Lundgren, 1976]; Lundgren \& Pointin, 1977]. One early proposal [Onsager, 1949] was that the temperature of such systems is negative, although others [Berdichevsky, et al., 1991] later showed that this is unnecessary if a different definition of the temperature is adopted.

In this paper we present the results of numerical experiments in which we produced interesting nontrivial collective motions of small populations of point vortices confined in a square box by judiciously selecting the coupling between vortex pairs. We are able to produce motions that are highly ordered, motions that are quasiperiodic, motions that are completely stable or exactly periodic, motions in which the vortices divide into two or more groups, and motions that are nearly, or completely, chaotic. In addition, we find that some systems exhibit spontaneous switching between several quasi-stable motions. This intermittent switching depends on the pairwise couplings, on the box size, and on the step size in the numerical integration. 


\section{SYSTEMS OF POINT VORTICES}

A collection of point vortices is defined by the Hamiltonian

$$
\begin{gathered}
H=\sum_{i \neq j} K_{i j} \log \left(D_{i j}\right) \\
D_{i j}=\sqrt{\left(x_{i}-x_{j}\right)^{2}+\left(y_{i}-y_{j}\right)^{2}}
\end{gathered}
$$

where $\left\{x_{i}, y_{i}\right\}$ are the canonical coordinates and momenta (which may be considered simply cartesian coordinates of the vortex), and $\mathrm{K}_{\mathrm{ij}}$ are arbitrarily selected circulations. From $\mathrm{H}$ we find the equations of motion:

$$
\begin{aligned}
\frac{d x_{i}}{d t} & =\sum_{j \neq i}^{N} K_{i j} \frac{\left(y_{i}-y_{j}\right)}{D_{i j}} \\
\frac{d y_{i}}{d t} & =-\sum_{j \neq i}^{N} K_{i j} \frac{\left(x_{i}-x_{j}\right)}{D_{i j}}
\end{aligned}
$$

In order to reduce the number of free parameters, we arbitrarily adopt the following constraint: The $\mathrm{N}$ vortices will be identified as either male $(\mathrm{M})$ or female (F). A population of $n M$ and $m F$ vortices will be written $P=\{n M+m F\}$. The NxN matrix $\mathrm{K}_{\mathrm{ij}}$ is taken to have only 4 independent values:

$$
\mathrm{K}_{\mathrm{ij}}=\mathrm{S}_{1} \delta_{\mathrm{iM}} \delta_{\mathrm{jM}}+\mathrm{S}_{2} \delta_{\mathrm{iF}} \delta_{\mathrm{jM}}+\mathrm{S}_{3} \delta \mathrm{i}_{\mathrm{M}} \delta \mathrm{j}_{\mathrm{F}}+\mathrm{S}_{4} \delta \mathrm{i}_{\mathrm{F}} \delta \mathrm{j}_{\mathrm{F}}
$$

For instance, a population of $P=\{2 M+3 F\}$ vortices will have $K_{i j}$ given by

\begin{tabular}{l|lllll}
$\mathrm{i} \backslash \mathrm{j}$ & $\mathrm{M}$ & $\mathrm{M}$ & $\mathrm{F}$ & $\mathrm{F}$ & $\mathrm{F}$ \\
\hline $\mathrm{M}$ & $\ldots$ & $\mathrm{S}_{1}$ & $\mathrm{~S}_{2}$ & $\mathrm{~S}_{2}$ & $\mathrm{~S}_{2}$ \\
$\mathrm{M}$ & $\mathrm{S}_{1}$ & $\ldots$ & $\mathrm{S}_{2}$ & $\mathrm{~S}_{2}$ & $\mathrm{~S}_{2}$ \\
$\mathrm{~F}$ & $\mathrm{~S}_{3}$ & $\mathrm{~S}_{3}$ & $\ldots$ & $\mathrm{S}_{4}$ & $\mathrm{~S}_{4}$ \\
$\mathrm{~F}$ & $\mathrm{~S}_{3}$ & $\mathrm{~S}_{3}$ & $\mathrm{~S}_{4}$ & $\ldots$ & $\mathrm{S}_{4}$ \\
$\mathrm{~F}$ & $\mathrm{~S}_{3}$ & $\mathrm{~S}_{3}$ & $\mathrm{~S}_{4}$ & $\mathrm{~S}_{4}$ & $\ldots$
\end{tabular}

The vectors $P=\{n M+m F\}$ and $S=\left\{S_{1}, S_{2}, S_{3}, S_{4}\right\}$ are sufficient to define the system. We emphasize that this is an arbitrary special case; $\mathrm{K}_{\mathrm{ij}}$ actually allows 
$\mathrm{N}^{2}$-N different values. $\mathrm{K}_{\mathrm{ii}}$ is not relevant, since vortices have no self-interaction.

Typical motions of small populations are shown in Figure 1, using circulations $S=\{1,-1,-1,1\}$. These steady motions are achieved in free space independent of initial configuration, often after lengthy transients, in the limit of zero step size.

In order to visualize motions that appear transiently on the computer screen, we display the positions of each vortex over some interval (typically the last 100 steps). To the extent that the step size is small, these patterns appear as relatively smooth trajectories. If the motion is periodic, the patterns appear as simple geometric figures. If the motion is smooth but chaotic, the trajectories appear as wandering curves. On the computer screen, these trajectories appear as rotating solids and worms.

Figure 2 shows typical time-integrated images of sets of vortices for systems similar to those of Fig. 1. These rosette patterns are a well-known representation of vortex systems.

The representation of the trajectories as time-integrated images is more than convenient; it converts the set of rapidly moving points into slowly moving geometric figures. These figures take on a meaning, or "life" independent of the point vortices themselves. The patterns appear on the screen as "organisms" that sometimes move, interact, evolve, appear, and disappear. Sometimes one pattern will "ingest" another, sometimes "disintegrate" another. Thus, we refer to these patterns as metastable states. While the underlying reality is the motion of the point vortices, it is the metastable patterns that we find interesting. In the rest of this paper, therefore, we will only present these images.

\section{VORTEX HEATING}

The hamiltonian given above is constant, hence the rosette patterns remain confined within a finite region determined by the total energy. If, however, we constantly add energy, the trajectories expand without limit. The addition of energy can be simulated very simply by taking finite step sizes in the numerical integration of the equations of motion. This process is roughly equivalent to "heating" the vortices.

Figure 3 shows a single heated vortex circulating around a fixed center. The system is $P=\{1 M+1 F\}, S=\{0,0,-1,0\}$. The $M$ vortex follows an expanding spiral pattern that would continue without limit in the absence of a constraint. 


\section{WALL STABILIZATION}

We can stop the outward spiral motion of a heated vortex by positioning a hard wall in its path. Computationally, this can be done very simply by repositioning an impinging vortex slightly away from the wall. Physically this is equivalent to giving the vortex an inward impulse when it strikes the wall, a process roughly equivalent to a stochastic "cooling" of the vortices.

Figure 4 shows the wall confinement of a single vortex circulating around a fixed center. When it hits the wall, it receives an inward kick that puts it nearly on the same trajectory as the previous circuit. Thus, it approaches the wall again on nearly the same trajectory, and finds itself nearly trapped, or stabilized, on the wall.

If the cooling exactly compensates for the heating, a vortex will be stabilized on the wall. While it was not obvious a priori that the numerical processes we used to simulate heating and wall cooling can exactly compensate, we found that wall stabilization is in fact relatively easy to obtain. In some cases, the trajectories, and therefore the time-integrated patterns, are absolutely stable. In others, the equilibrium is not perfect, and the pattern "walks" or "slides" along the wall. We also observe the sudden total chaotic disintegration of a seemingly stabilized pattern.

Figure 5 shows vortex confinement in a box. The conditions are very similar to Fig. 4 . Here we show the effect of increasing the heating rate by increasing $\mathrm{S}_{3}$. For small $S_{3}$, the trajectories are stabilized on one wall, forming a ring with a slight flattening. As $\mathrm{S}_{3}$ increases, the pattern expands, and becomes flattened against the wall, like a rubber tire against a road. At very large $S_{3}$, the pattern expands like a balloon to fill the box.

Figure 6 shows the transient associated with wall stabilization. A set of fixed $F$ vortices provide guide centers for $10 \mathrm{M}$ vortices that circulate around them. Initially the vortices were randomly positioned; vortices near a center made local spiral trajectories around those centers. As the vortices expanded due to the heating, they began to enclose additional centers, until eventually all vortices were enclosing all centers. The pattern was permanently stabilized by the short flat segment at lower right, and circulated forever on the track-like pattern shown in Fig. 6(c). 


\section{SWITCHING}

It is apparent from Fig. 6 that a vortex can switch suddenly from one trajectory to another. Typically this might occur if the vortex is coupled nearly equally to more than one center: a small amount of heating or cooling will cause the switch. Figure 7 shows this process clearly. In this case, a set of vortices circulate $\mathrm{CW}$ in the vicinity of 2 fixed centers, each of which is near a wall. Initially, the vortices are near the left center and away from the wall; they circulate in nearly circular orbits. As they are heated, the orbits expand, and begin to get distorted under the influence of the right-hand center. One by one, the vortices break away to the right and pass near the right-hand center. Their velocity is so high that they impact on the wall, experiencing cooling and becoming stabilized on the right-hand center. Eventually, all vortices have switched to the right-hand center, where they circulate forever. Seen as timeintegrated patterns, the effect is that the circular pattern jumps suddenly from one location to another.

The motion of vortices around the fixed centers is easily visualized as a particle moving in a potential well. Figure 8 shows this for the two-center system of Fig. 7. A vortex initially placed in the left-hand well rises due to the heating, eventually overcoming the central hump and reaching the right-hand well. The cooling at the wall drops it below the energy necessary to pass over the barrier, and it is trapped.

Intermittent switching can occur if the stable pattern has some noise. For instance, the stabilized pattern of Fig. 6(c) has a width that fluctuates but never vanishes. If it happened that this pattern passed between two attracting centers of comparable strength, we would expect the trajectories to sometimes follow one pattern, sometimes another. To the extent that the pattern is truly chaotic, this switching will be truly intermittent. Figure 9 shows this effect for system of 5 attracting centers, one of which is separated from the other 4 . At relatively low energy, the moving vortices circulate around the 4 centers, stabilized by the wall (Fig. 9(a)). If we increase the heating rate (Fig. 9(c), it switches to include the outlying center, just as in Fig. 7. However, this time the trajectories switch intermittently between the inner pattern and the outer pattern. As the heating increases further (Fig. 9(d)), only the outer pattern occurs.

Somewhat surprisingly, within a very narrow region of higher heating rate, 
the pattern shifts back to include the inner trajectories: in Fig. 9(e) the intermittent double pattern is again obtained. Alternatively, we could view this as a small region (d) of greater stability within the larger region (c)-(e) of intermittency. The cause of this stability is the quantized nature of the wall cooling in the present numerical technique. Whenever the vortex hits the wall, it bounces back a fixed distance, then drifts to the side and approaches the wall again, bouncing again, etc. By the time it leaves the wall, it has made a certain number of bounces, and is launched into a certain trajectory. A small increment of heating may change the launching trajectory only slightly, so the complete pattern will be only slightly perturbed. However, if a small increment results in the addition of another bounce, the vortex is launched onto quite a different trajectory, eventually switching to a different branch in the pattern. In the limit that the cooling impulses are infinitesimal, the trajectories are simply flattened against the wall, and the switching from one pattern to another will be sharp and noise-free.

\section{COMPLEX SYSTEMS}

Having presented the fundamental dynamical behavior of individual confined heated vortices, we now examine examples of systems that are more complex. We will find that they typically establish a metastable state that has a characteristic pattern and persists for a relatively long time (many vortex orbits), but then may suddenly switch to another metastable pattern.

Figure 10 shows a variety of quasi-stable motions for a system of 2 male and 2 female vortices. The system was $\mathrm{P}=\{2 \mathrm{M}+2 \mathrm{~F}\}, \mathrm{S}=\left\{1.3,-1.4, \mathrm{~S}_{3},-1.4\right\}$, and $\mathrm{S}_{3}$ is varied from -20 to +20 .

The patterns seen in Fig. 10 include four motions that appear repeatedly:

(1) counter-rotating concentric rings or lobed rosettes

(2) localized wall-stabilized chaotic nests that include the entire population

(3) separated wall-stabilized nests that include subsets of the population

(4) wall-confined but unstabilized chaotic swarms.

Each of these patterns was relatively stable; it did not spontaneously switch to another pattern. Although each pattern constantly changed in detail, the appearance of the screen was roughly the same forever. For $S_{3}<0$, the motions can be described as the females circulating within the box and the males confined near the center of the box. As soon as $S_{3}>0$, a new phenomenon occurs: the males 
and females separate into two relatively localized "nests," with one (the males) stabilized in one corner and the other stabilized on the wall. At low $S_{3}$, the female nest repeatedly approaches the male nest, resulting in temporary disruption of the patterns. With increasing $S_{3}$, the distance between these nests increases, but as $S_{3}$ increases above 1.4, the male nest begins to attack the female nest, eventually driving the system into complete chaos.

Although most of the patterns in Fig. 10 are metastable, some of them are unstable, and spontaneously go into oscillation. Figure 11 shows one such example. Initially, with $\mathrm{S}_{3}=1.3$, the $2 \mathrm{M}$ and $2 \mathrm{~F}$ nests were stabilized in opposing corners. By altering $S_{3}$, it was possible to induce the female nest to emerge from the corner and approach the male nest. Then $S_{3}$ was reset to 1.3. The result was that the $2 \mathrm{~F}$ nest hovered "nervously" for a long time near the $2 \mathrm{M}$ nest, then suddenly disintegrated by changing to a jet of vortices directed at the $2 \mathrm{M}$ nest. The jet caused considerable agitation of the $2 \mathrm{M}$ nest, which ejected the $\mathrm{F}$ vortices one by one. Stabilizing on the wall, the F vortices slowly reformed into a new nest, and the process repeated. This oscillation appeared to persist forever. The period was relatively well-defined.

A more complex system that oscillates between a highly ordered metastable state and an almost completely chaotic state is shown. in Figure 12. The system was $\mathbf{P}=\{4 \mathrm{M}+4 \mathrm{~F}\}, \mathbf{S}=\{1,-1,1,-1\}$. The series of frames show the pattern at roughly equal intervals. About half the frames show two counter-rotating rings, the $4 \mathrm{~F}$ vortices circulating around the $4 \mathrm{M}$ vortices, while the other frames show almost completely chaotic wandering (perhaps with slight suggestions of internal circulation).

Figure 13 shows a system that switched intermittently between four metastable states. This system was $\mathbf{P}=\{3 \mathrm{M}+2 \mathrm{~F}\}$ with $\mathrm{S}=\{1,-1,1,1\}$. Each of the four frames shows a typical pattern that persisted for an unpredictable time, whereupon it would switch suddenly to one of the others. This was not an oscillator; there was no obvious pattern to the sequence of motions. 


\section{WALL-INDUCED STABILIZATION}

The stabilization shown in the previous examples is dynamic-if the wall is removed, the patterns resume their expansion. However, we have also found cases of wall-induced stabilization: patterns remain stable after the wall is removed. Figure 14 shows an example. The system consisted of $9 \mathrm{M}$ vortices $P=\{9 \mathrm{M}\}$, with circulations $\mathbf{S}=\{r, \ldots, \ldots, \ldots\}$, where $\mathrm{r}$ indicates a pseudorandom number $(0,1)$. The sequence of figures shows how the initially chaotic motion was stabilized by a smaller box, and remains completely stable after that box is removed. This pattern does not expand, although it is being numerically heated. The detailed mechanism by which this system becomes self-cooling is unknown.

\section{CONCLUSIONS}

The central result from this study is that wall stabilization of small populations of heated point vortices can produce to a variety of metastable states and collective motions, ranging from simple rigid body rotation to complete chaos. These patterns are not seen in adiabatic vortex systems.

There is some possible application of these observations to biological phenomena. Figure 15 shows two sequences of 5 frames in which two "nests" interact. In each case, $3 \mathrm{~F}$ vortices form a "nest" that slowly approaches a $2 \mathrm{M}$ nest stabilized in the corner. The sequence in Fig. 15(a) is reminiscent of the ingestion of a protozoan by another larger protozoan. The sequence in Fig. 14(b) is more interesting. It is known that certain cnidarians (sea anemones) such as Corynactis californica have very aggressive defense mechanisms [Morris, et al., 1980]. When approached by another anemone, Anthopleura elegantissima, Corynactis will extend a mesentary filament that stings the victim, causing its death and complete disintegration of the organism [Chao, 1975]. The process in Fig. 15(b), while not accurate in all these details, is broadly suggestive of such behavior.

The general character of these systems is worth generalizing. We expect any collection of objects to expand when heated. Confining the collection while maintaining heating is equivalent to cooling the objects at the walls. Thus, we obtain a dynamic equilibrium that will almost certainly be very different from the adiabatic equilibrium obtained without heating and cooling. A very general response of systems under these conditions is ordering: the system undergoes 
phase transitions that increase internal order. Examples are domain formation, crystallization, and coherent motion.

Based on the results of this paper, we therefore expect to find a wide range of new and interesting epi-phenomena associated with small populations of confined objects subject to heating. Because of their attractive individual dynamics, small populations of point vortices provide a very interesting system with which to study these phenomena.

\section{REFERENCES}

Arecchi, F. T., Giacomelli, G., Ramazza, P. L. \& Residori, S. [1991] "Vortices and defect statistics in two-dimensional optical chaos," Phys. Rev. Lett. 67, 3749. Aref, H. and Pomphrey; N. [1982] "Integrable and chaotic motions of four vortices. I. The case of identical vortices," Proc. Roy. Soc. Lond. A380, 359-387.

Aref, H. [1979] "Motion of three vortices," Phys. Fluids 22,393-400.

Berdichevsky, V, Kunin, I., and Hussain, F. [1991], "Negative temperature of vortex motion," Phys. Rev. A43, 2050-2051.

Busse, F. H. [1994] "Convection driven zonal flows and vortices in the major planets," Chaos 4(2),123-134.

Chao, C. [1975]. Inter-specific agression between three sympatric anemones, Anthopleura elegantissima, Metridium senile, and Corynactis california. Res. Paper Biol 175h, Hopkins Marine Sta., Stanford Univ., Pacific Grove, CA.

Glanz, J. [1994] , "Physicists find windows of opportunity in plasmas," Science 266,1638-39.

Harada, K., Matsuda, T. Bonevich, J., Igarashi, M., Kondo, S., Pozzi, G., Kawabe, U \& Tonomura, A. [1992] "Real-time observation of vortex lattices in a superconductor by electron microscopy," Nature 360,51-53.

Hopfinger, E. J. \& van Heijst, G. J. F. [1993] "Vortices in rotating fluids," Ann. Rev. Fluid Mech. 25, 241-289.

Jeffery, M. \& D'Angelo, E. J. [1994] “Optical Vortex Interactions in Lasers," Intl J. Bifurcation and Chaos 4(3), 675-691.

Kunin, I. A., Hussain, F., Zhou, X, and Prishepione, S. J. [1992] "Centroidal frames in dynamical systems. I. Point vortices," Proc. R. Soc. Lond. A439, 441463.

Lundgren, T. S. and Pointin, Y. B. [1977] "Non-gaussiar probability distributions for a vortex fluid," Phys. Fluids 20,356-363. 
McWilliams, J. C. \& Weiss, J. B. [1994] "Anisotropic geophysical vortices," Chaos 4(2),305-311.

Morris, R. H., Abbott, D. P., and Haderlie, E. C. [1980]. Intertidal Invertebrates of California. Stanford Univ. Press.

Onsager, L. [1949] "Statistical Hydrodynamics," Nuovo Cim. (suppl.) 6,279-287.

Pointin, Y. B. and Lundgren, T. S., [1976] "Statistical mechanics of two-

dimensional vortices in a bounded container," Phys. Fluids 19,1459-1470.

Sutyrin, G. G. \& Yushina, I. G. [1986] "Evolution of solitary vortices in a rotating fluid," Fluid Dyn. 21(4),550-556.

Sutyrin, G. G. [1994] “Long-lived planetary vortices and their evolution:

Conservative intermediate geostrophic model," Chaos 4(2),203-212.

van Heijst, G. J. F. \& Kloosterziel, R. C. [1989] "Tripolar vortices in a rotating fluid," Nature 338, 569-571. 


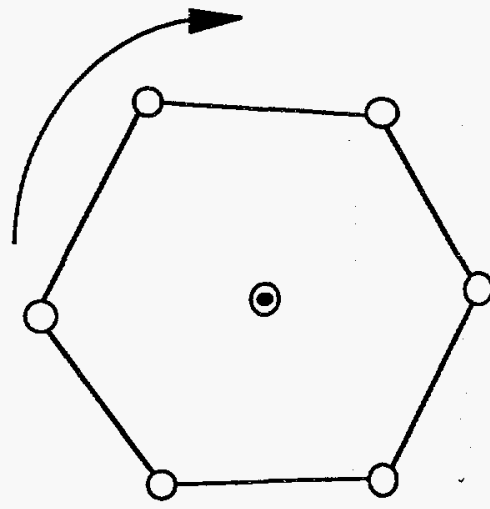

(a)

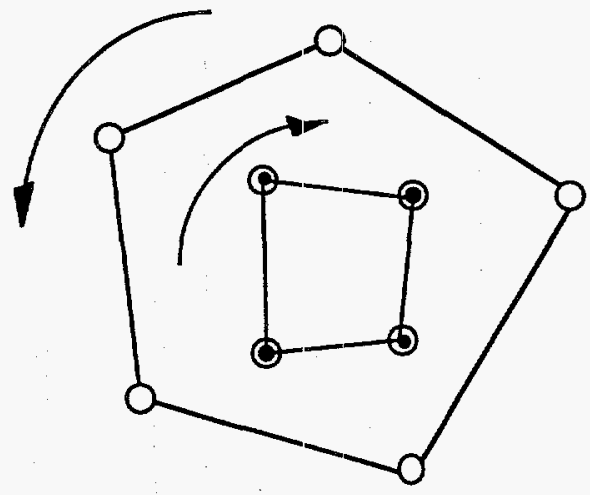

(b)

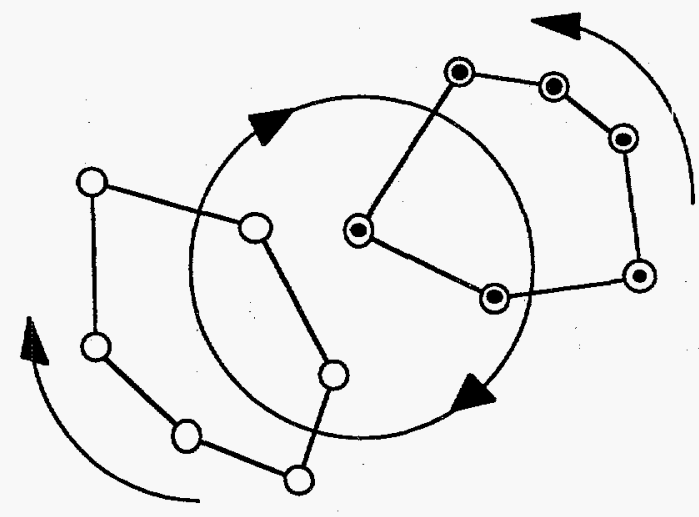

(c)

Figure 1 - Typical motions of several small populations of interacting point vortices. The populations include "male" (M) and "female" (F) vortices, as follows:

(a) $\mathrm{P}=\{1 \mathrm{M}+6 \mathrm{~F}\}, \mathrm{S}=\{1,-1,-1,1\}$

(b) $\mathbf{P}=\{4 \mathrm{M}+5 \mathrm{~F}\}, \quad S=\{1,-1,-1,1\}$

(c) $\mathbf{P}=\{6 \mathrm{M}+6 \mathrm{~F}\}, \mathbf{S}=\{1,-1,-1,1\}$ 


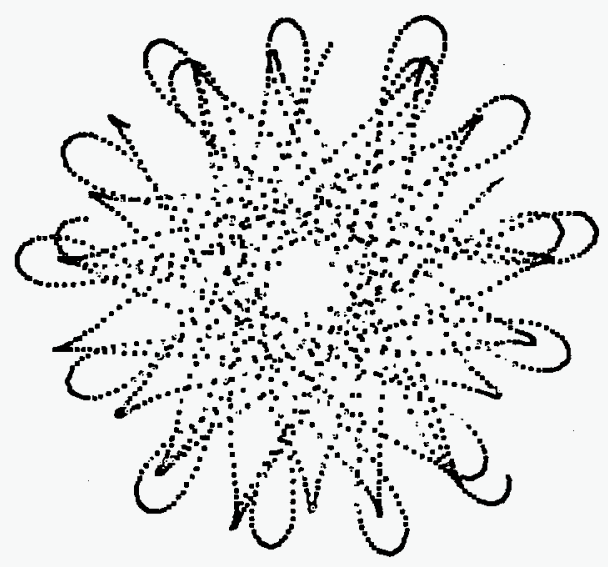

(a)

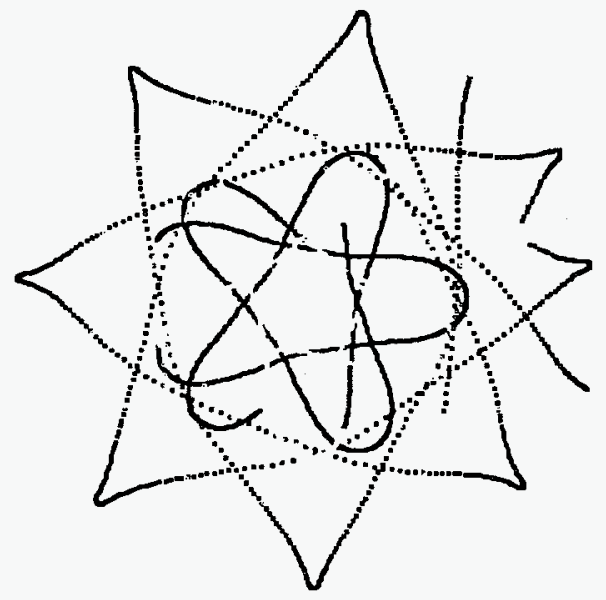

(b)

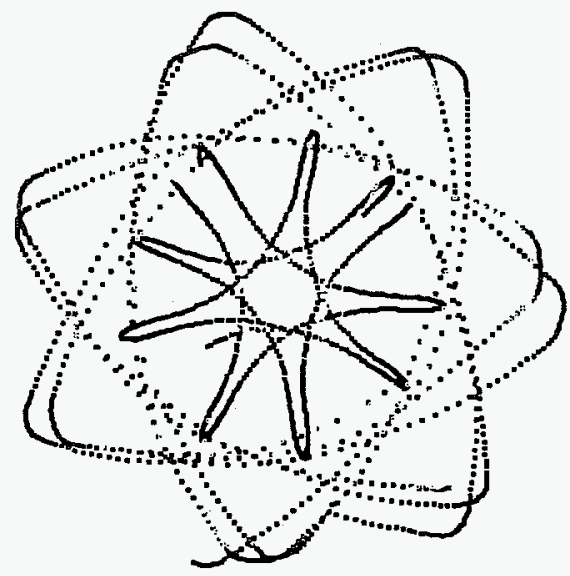

(c)

Figure 2 - Typical motions of several small populations of interacting point vortices. All have $\mathbf{P}=\{2 \mathrm{M}+6 \mathrm{~F}\}$
(a) $\mathrm{S}=\{0.6,-0.8,-0.8,1.1\}$
(b) $S=\{0.2,-0.4,-0.1,0.3\}$
(c) $\mathrm{S}=\{0.2,-0.7,-0.2,0.3\}$ 


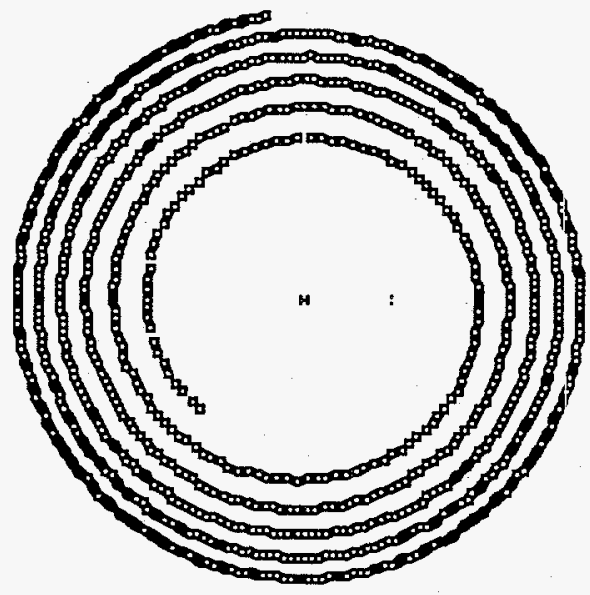

Figure 3 - Vortex heating. A single vortex spirals forever outward from a single fixed vortex. $\mathbf{P}=\{1 \mathrm{M}+1 \mathrm{~F}\}, \mathrm{S}=\{0,0,-0.2,0\}$. 


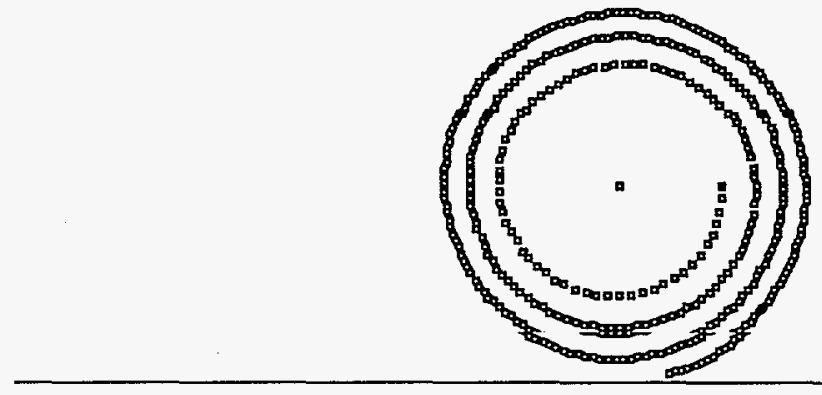

(a)

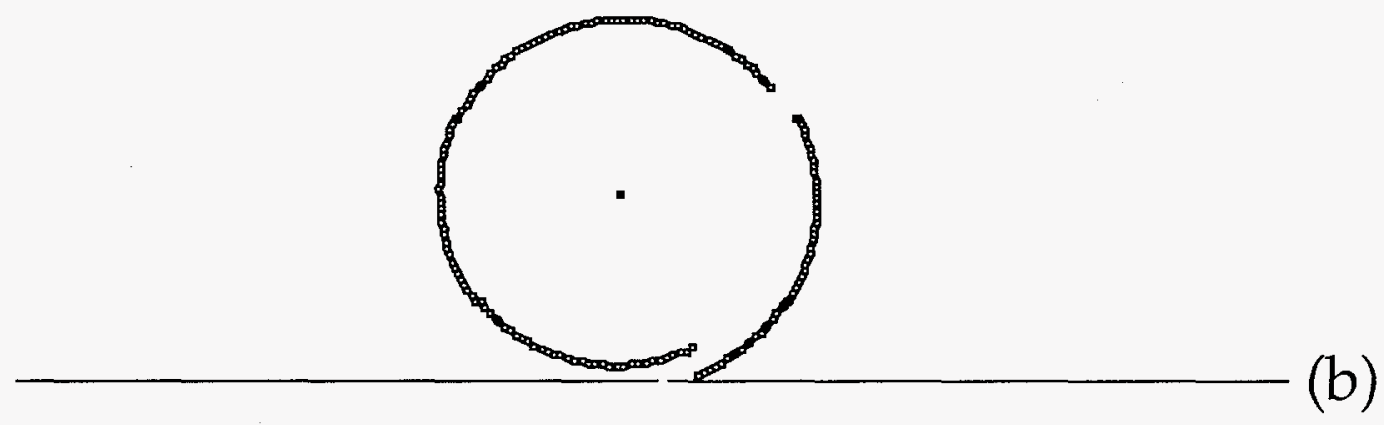

Figure 4 - Wall stabilization. (a) A heated vortex spirals toward a wall. (b) When it hits the wall, it is given an inward kick that compensates for the heating. $P=\{1 \mathrm{M}+1 \mathrm{~F}\}, \mathbf{S}=\{0,0,-$ $0.2,0\}$. 

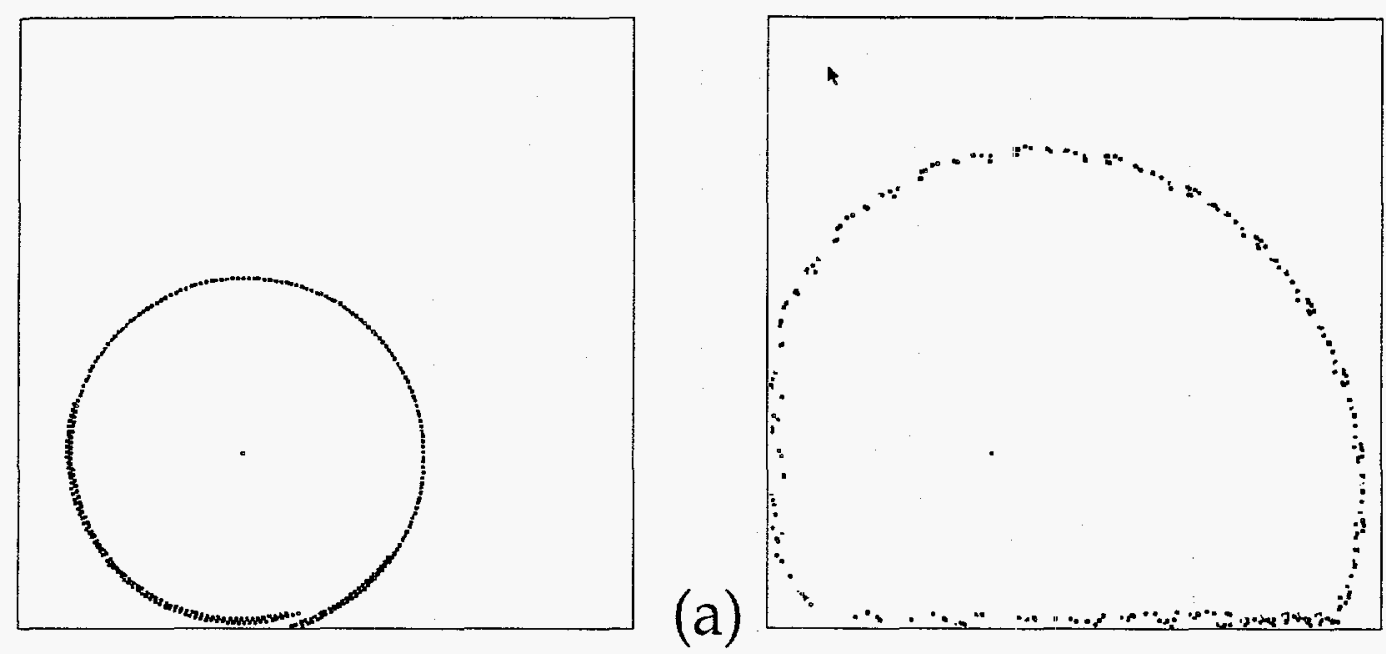

(c)
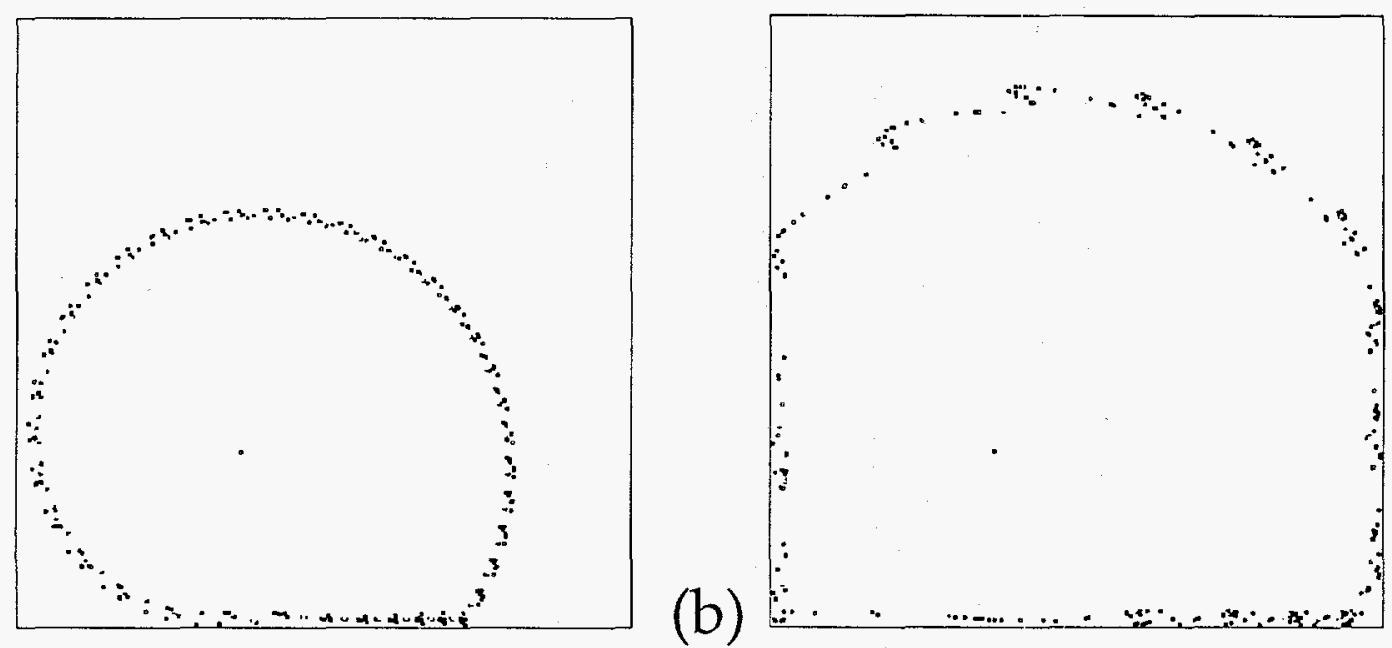

(d)

Figure 5 - Stabilization in a box. A single vortex circulates $\mathrm{CW}$ around a stationary $\mathrm{M}$ vortex. Within a small range of jitter, this pattern is stable forever. $\mathbf{P}=\{1 \mathrm{M}+1 \mathrm{~F}\}$.
(a) $S=\{\ldots, \ldots,-1, \ldots\}$
(b) $\mathrm{S}=\{\ldots, \ldots,-10, \ldots\}$
(c) $S=\{\ldots, \ldots,-30, \ldots\}$
(d) $S=\{\ldots, \ldots,-50, \ldots\}$

The ... parts of $\mathbf{S}$ do not enter. 


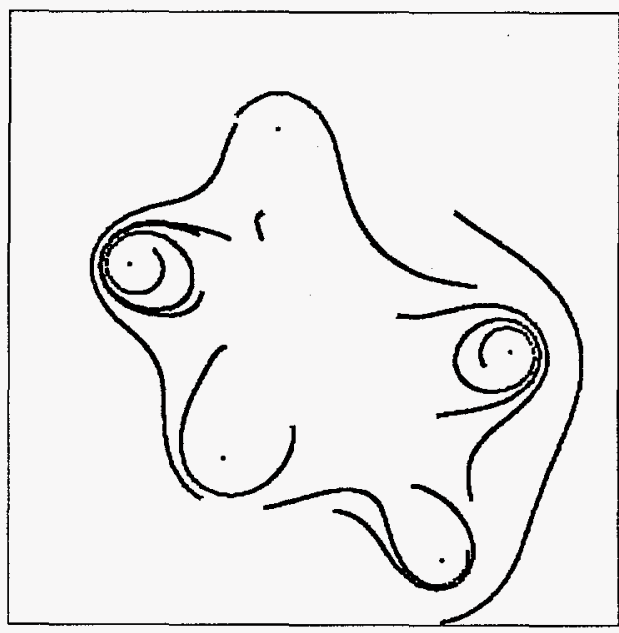

(a)

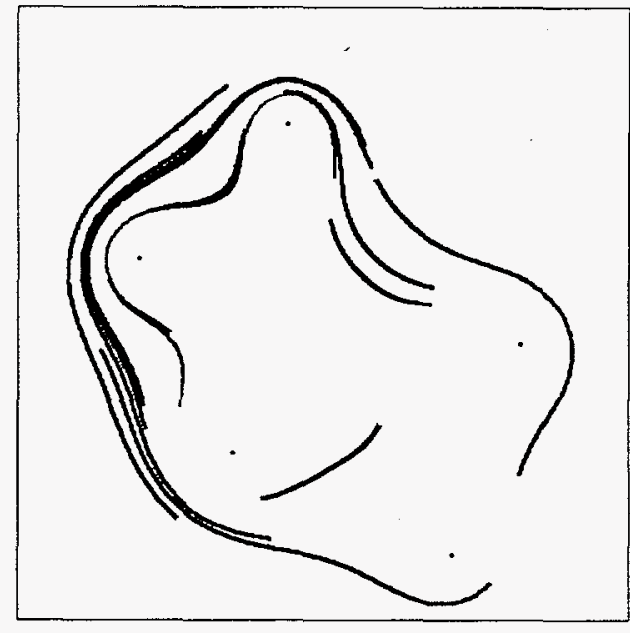

(b)

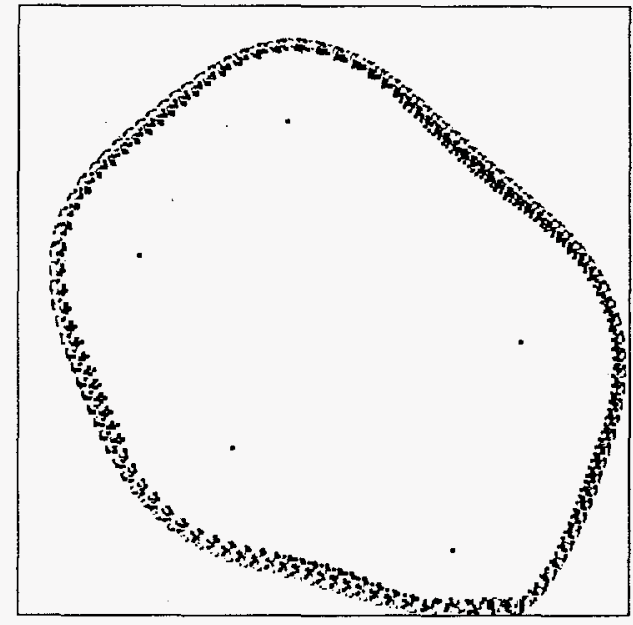

(c)

Figure 6-Approach to stablization. A set of $M$ vortices start at random positions in an arbitrary field of fixed $\mathrm{F}$ vortices. At large time they relax to a reasonably narrow circulating track. $\mathbf{P}=\{10 \mathrm{M}+5 \mathrm{~F}\}, \mathbf{S}=\{0,0,-0.1,0\}$. 

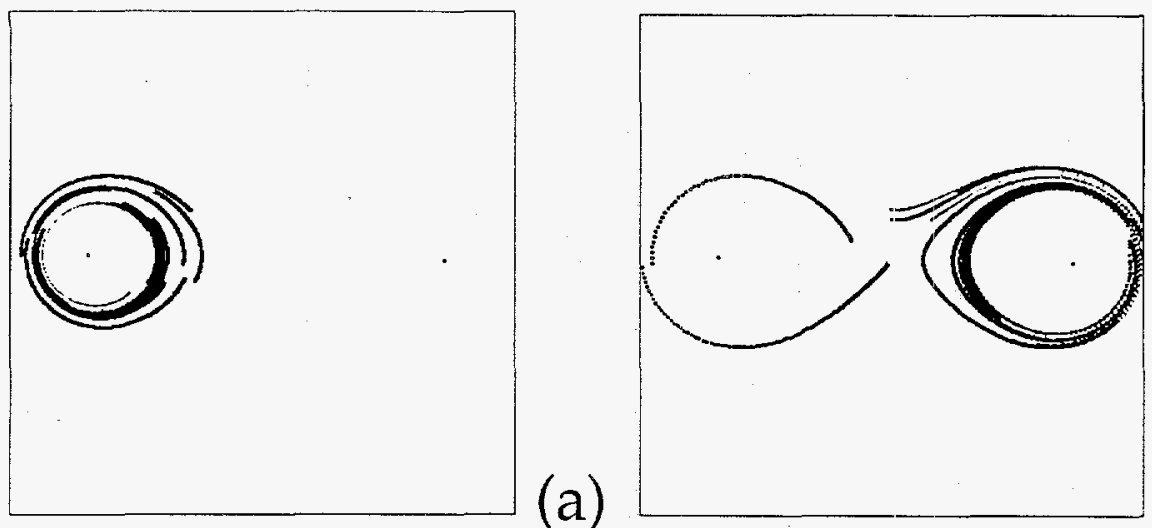

(d)
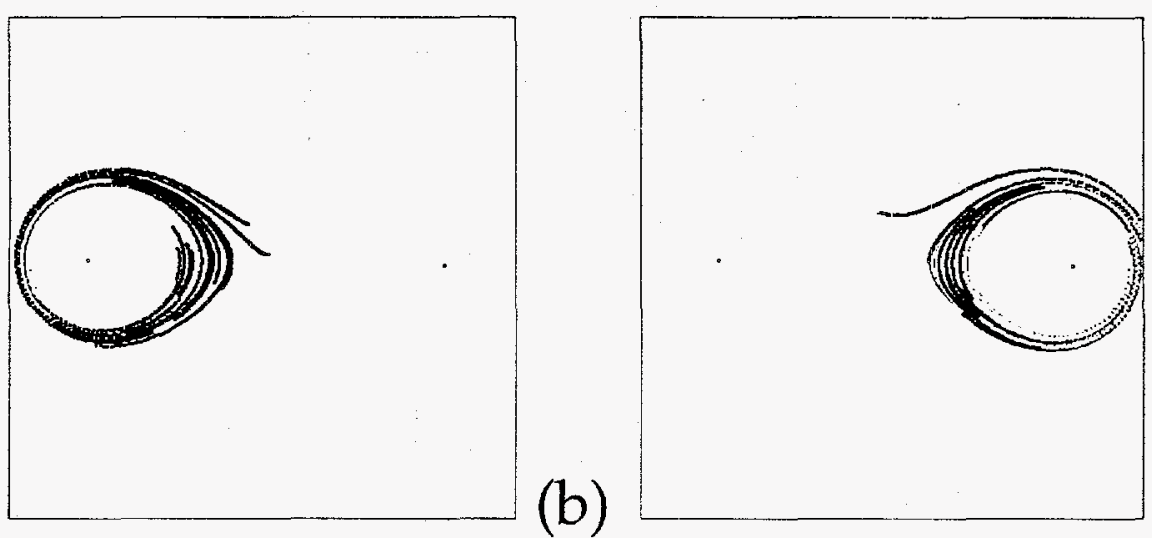

(e)

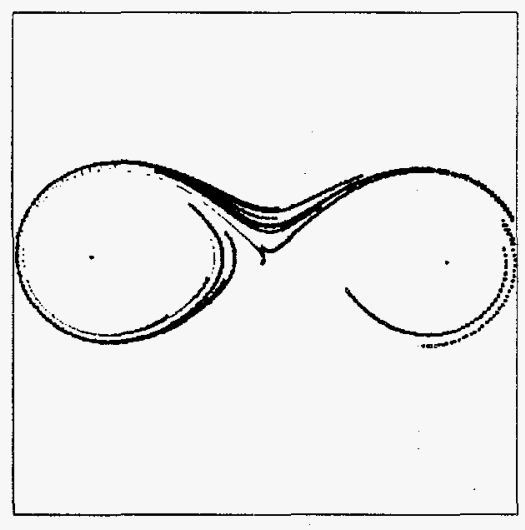

(c)

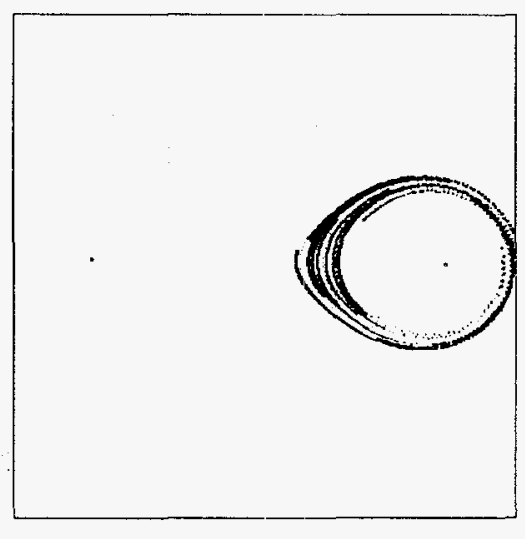

(f)

Figure 7 - Transient switching. A population of $10 \mathrm{M}$ heated vortices spends some time circulating around the left center, then spontaneously switches to the right one. Cooled by the wall, it never returns.

$P=\{10 M+5 F\}, S=\{0,0,-0.1,0\}$. 


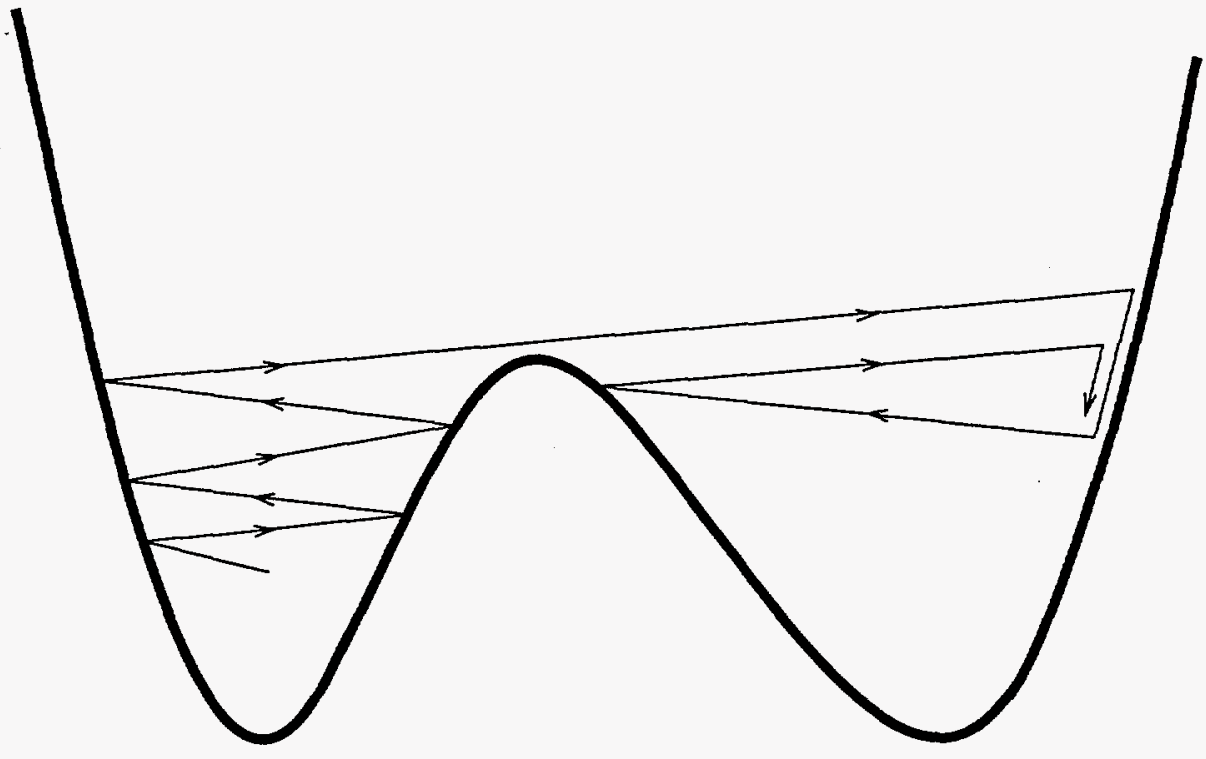

Figure 8 - Energy diagram for the system of Fig. 7. A vortex that starts in the lefthand well rises due to heating, eventually surmounting the central barrier. Cooling at the righthand wall traps the vortex in that well. 

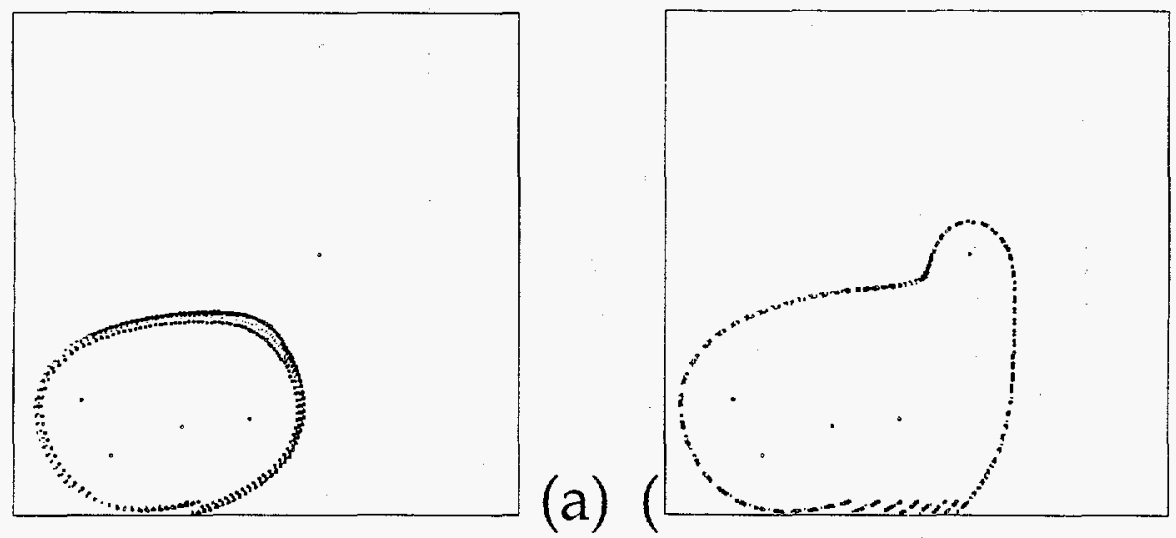

(d)

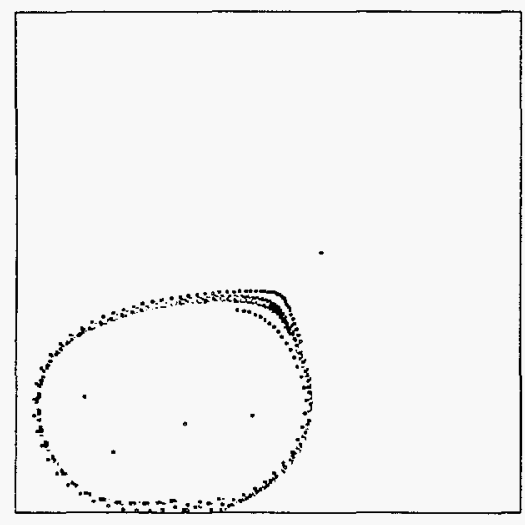

(b)

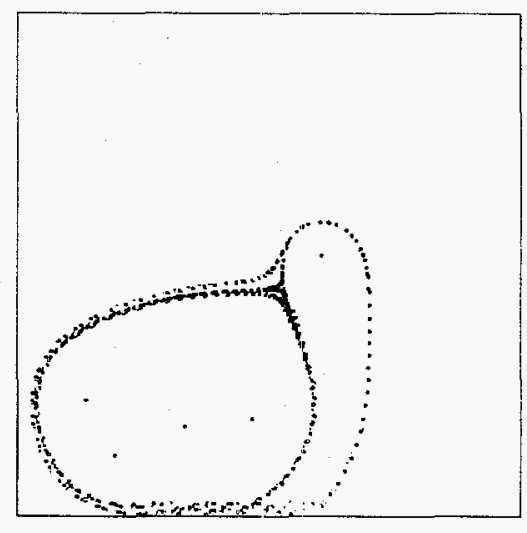

(e)

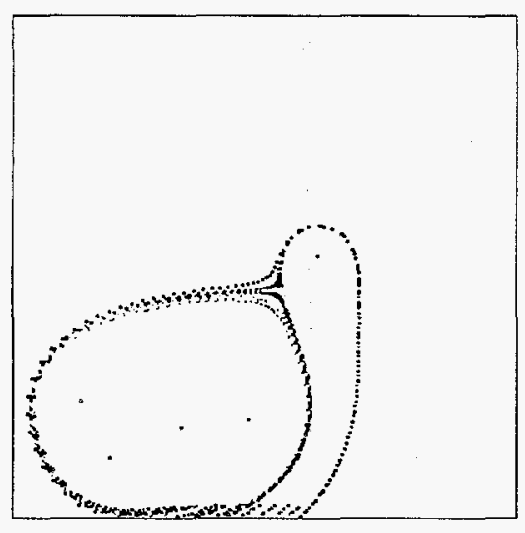

(c)

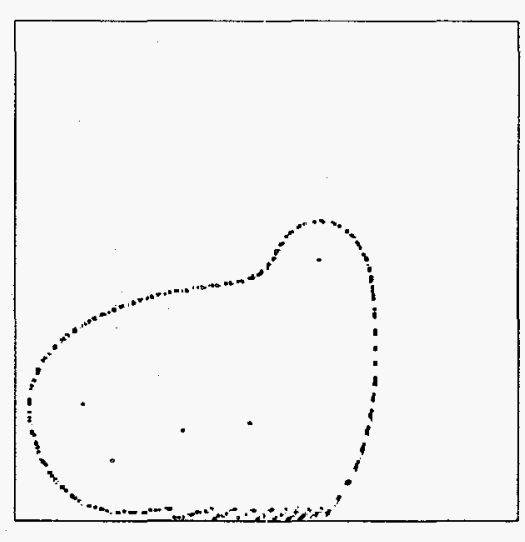

Figure 9 - Spontaneous switching. A wall-stabilized population of 5 vortices circulate in a field of 5 centers. At low leating (a)(b), the wall cooling keeps the circulation confined to a subset of $4 \mathrm{~F}$ vortices. With additional heating, the trajectories expand and switch intermittently between the two domains (a)-(e). Embedded within the switching range is an additional nonswitching range (d). $S=\left\{0,0, S_{3}, 0\right\}$; For (a)-(f), respectively, $S_{3}=-0.3,-0.6,-0.7$, $-0.8,-0.9$, and -1.2 . 

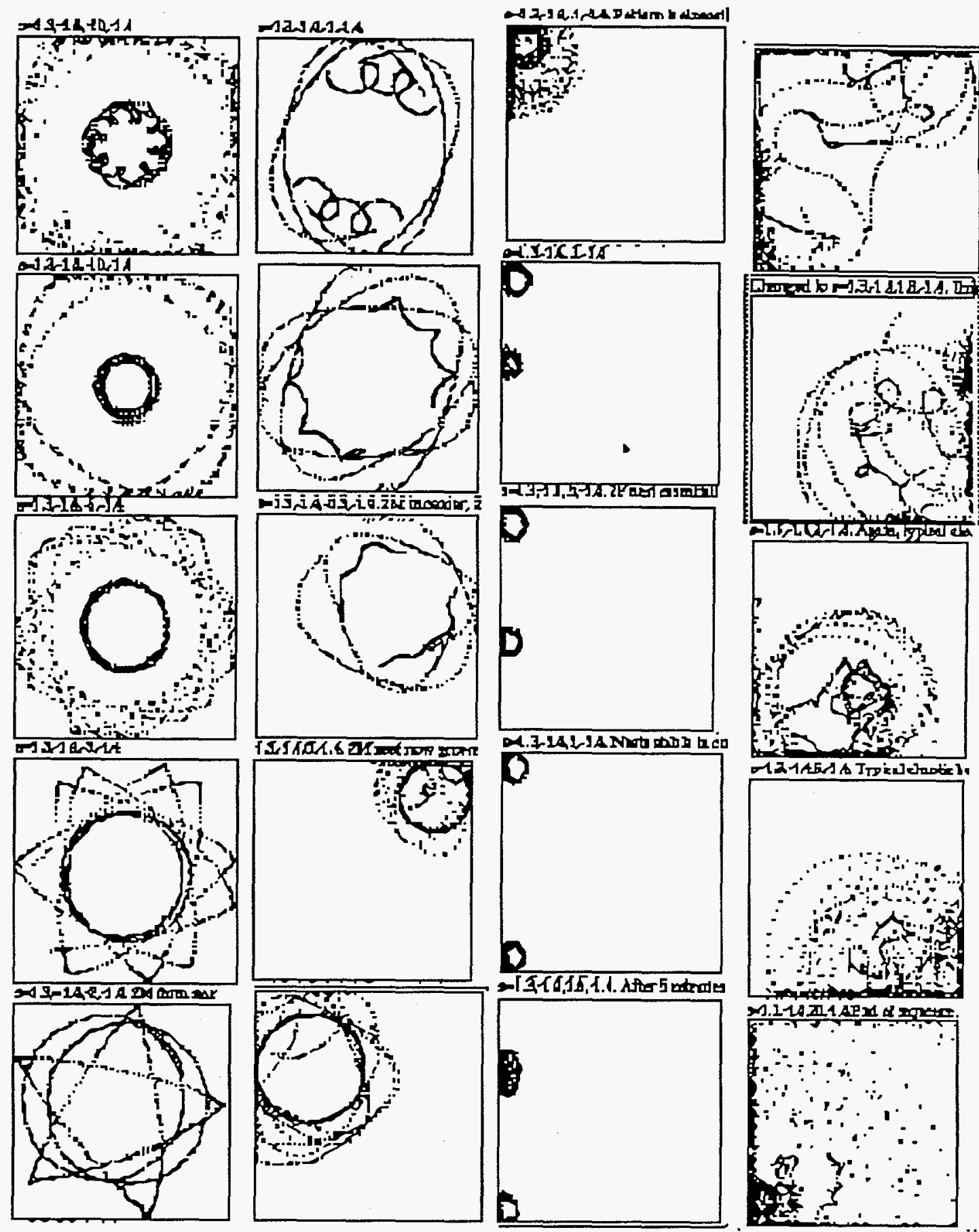

Figure 10 - Quasi-stable motions of 4 vortices, $P=\{2 \mathrm{M}+2 \mathrm{~F}\}$,

$S=\left\{1.3,-1.4, S_{3},-1.4\right\}$. The values of $S_{3}$ for the array are:

$\begin{array}{lrrr}-20 & -1 & +0.1 & \text { (later) }+1.6 \\ -10 & -0.5 & +0.3 & +1.8 \\ -5 & -0.3 & +0.5 & +3 \\ -3 & 0 & +1.0 & +5 \\ -2 & \text { (later) } 0 & +1.6 & +20\end{array}$



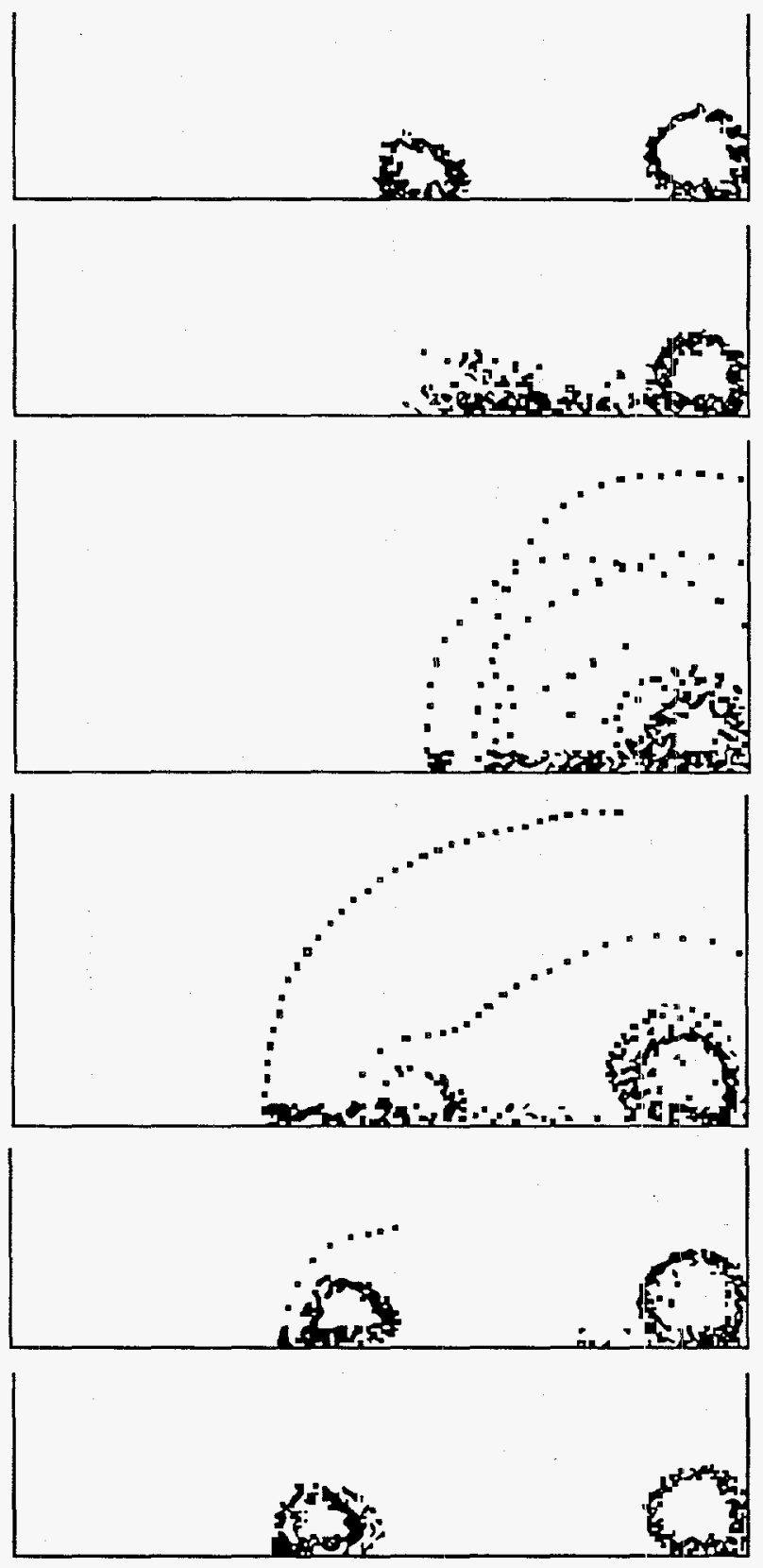

Figure 11 - Oscillation of 4 vortices confined in a box (sequence top to bottom). The population is $\mathbf{P}=\{2 \mathrm{M}+2 \mathrm{~F}\}$, with circulations $S=\{1.3,-1.4,1.3,-1.4\}$. The $2 \mathrm{M}$ vortices are in the right "nest" stabilized in the corner, and the $2 \mathrm{~F}$ vortices are in the left one. The $2 F$ slowly approach the $2 \mathrm{M}$ vortices, self-destruct, and reform for repeated cycles. 


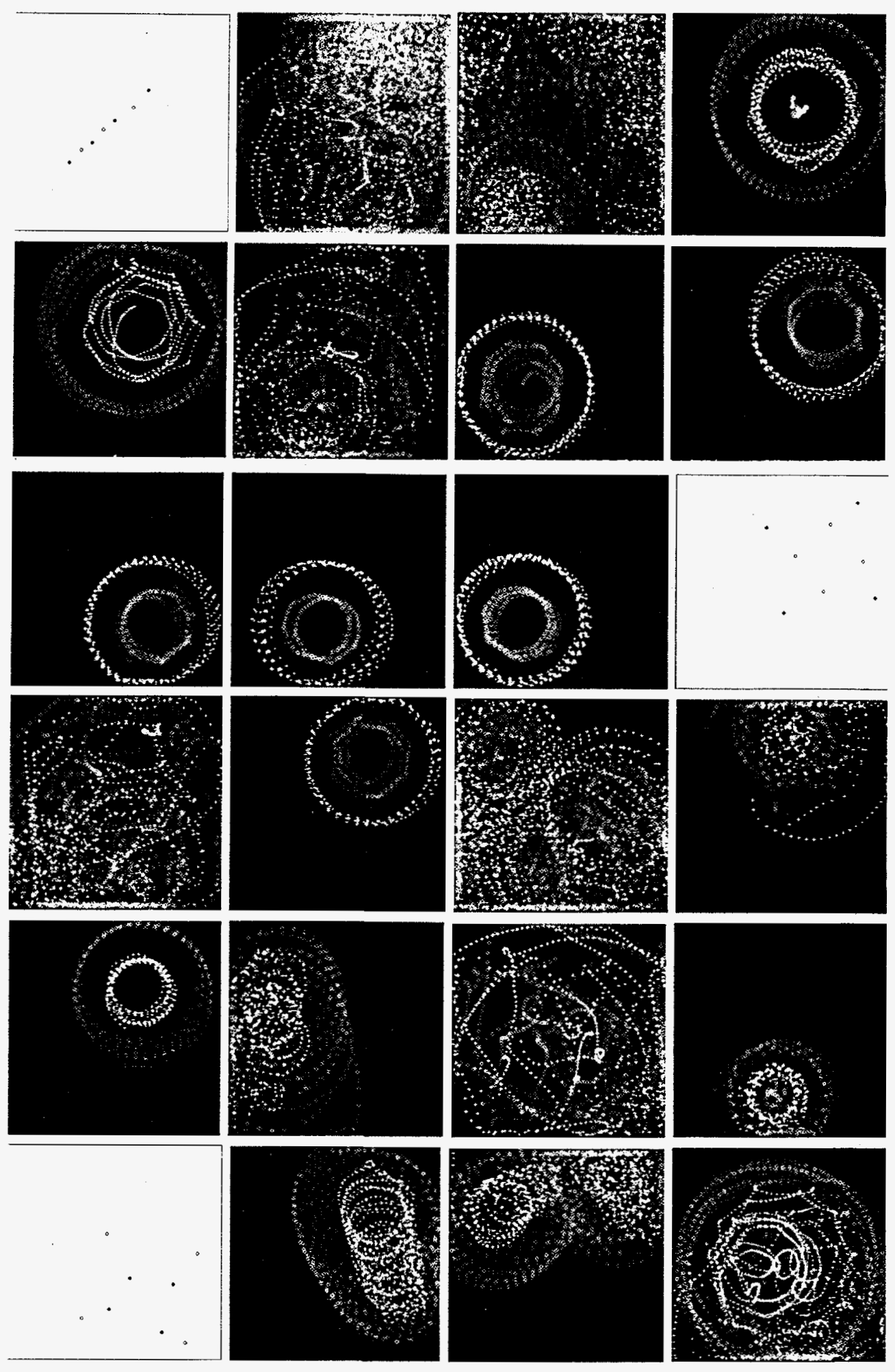

Figure 12 - Spontaneous intermittent switching between 2 meta-stable states. $\mathbf{P}=\{4 \mathrm{M}+4 \mathrm{~F}\}, \mathbf{S}=\{1,-1,1,1\}$. The frames are sequenced across, then down, i.e., normal book layout. The white frames show instantaneous positions of the vortices. 

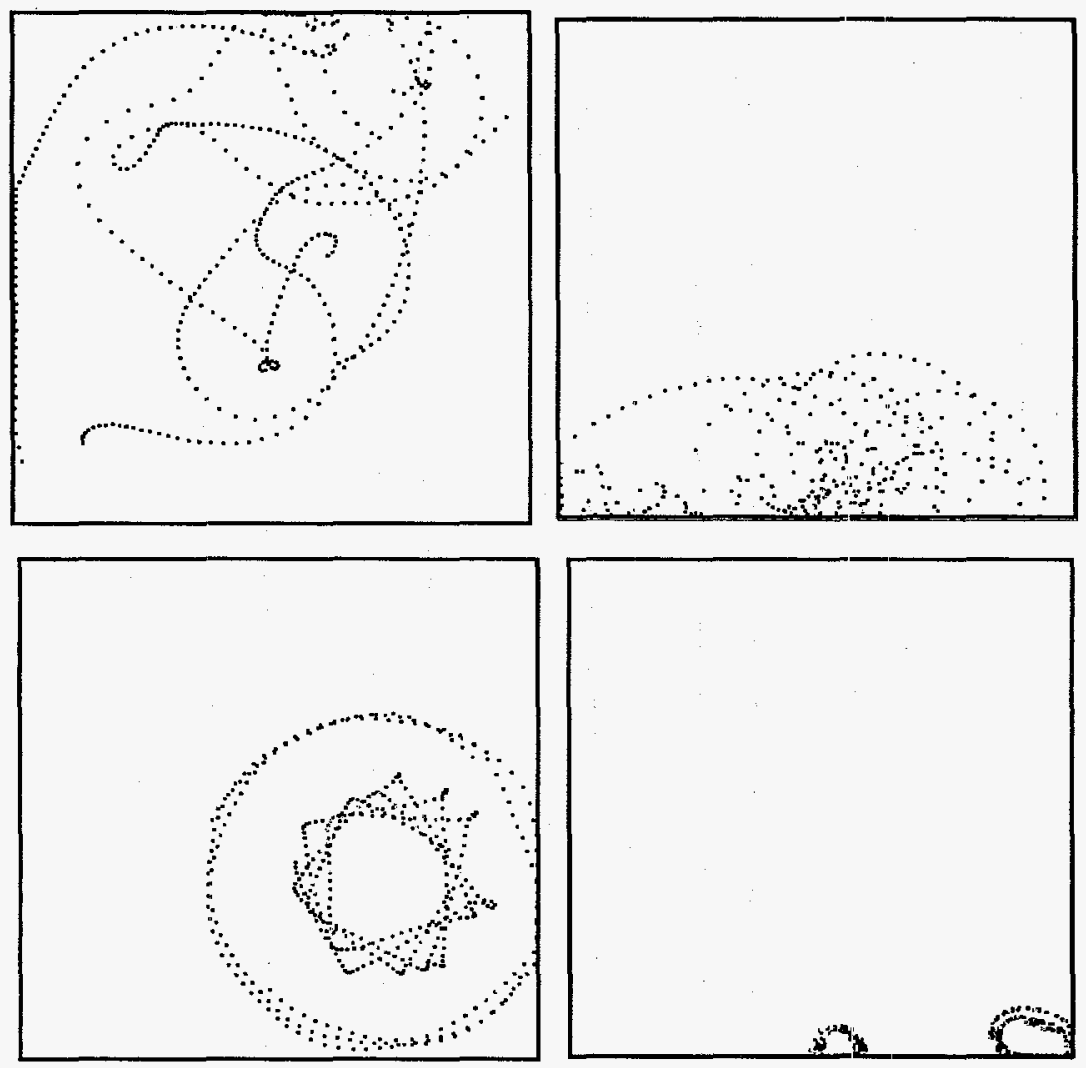

Figure 13 - Intermittent switching between 4 metastable states. $\mathbf{P}=\{3 \mathrm{M}+2 \mathrm{~F}\}, \mathbf{S}=\{1,-1,1,1\}$. This system produced a series of frames similar to Fig. 10, with random sequencing among these four metastable motions. 

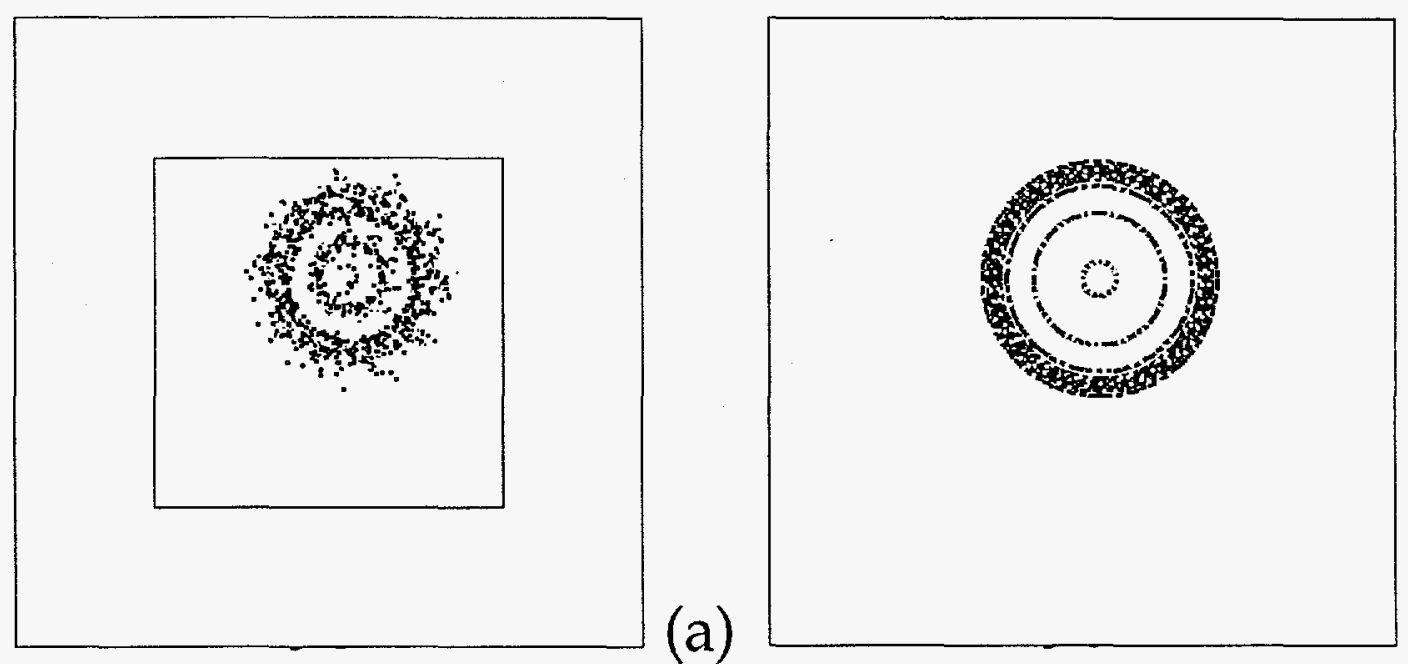

(c)

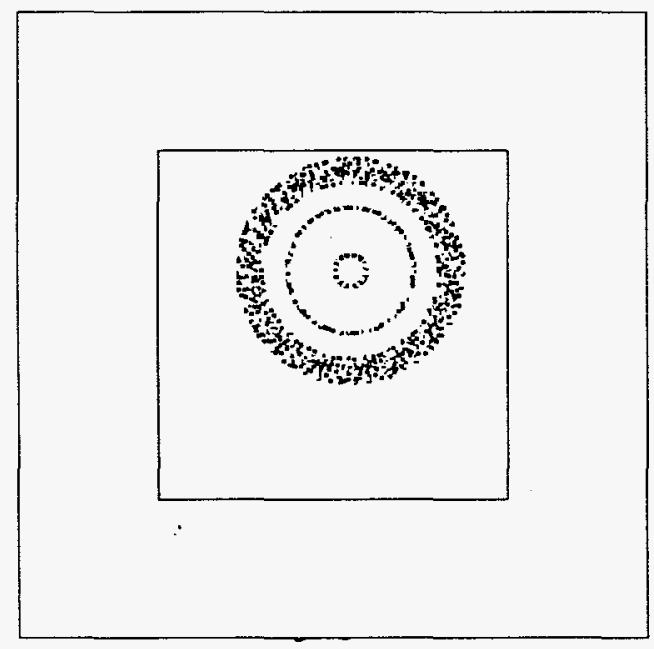

(b)

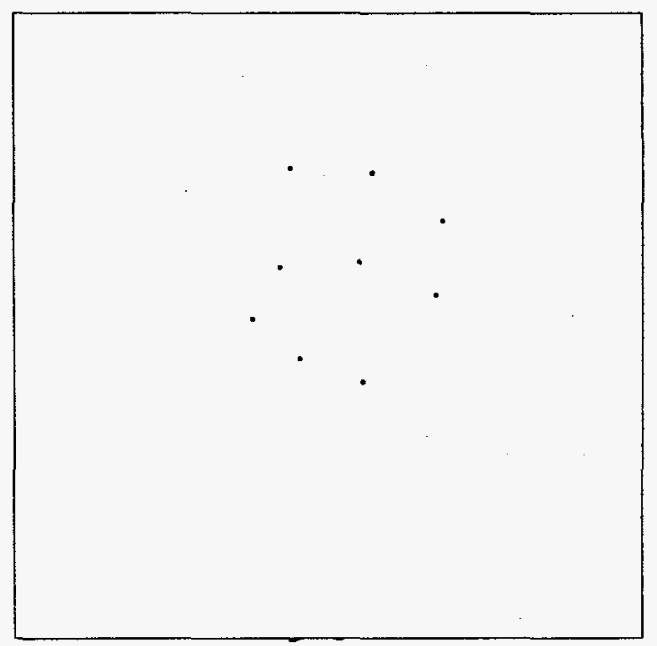

Figure 14 - Wall-induced stabilization. The population is 9 vortices $\mathbf{P}=\{9 \mathrm{M}\}$, with $\mathbf{S}=\{\mathrm{r}, \ldots, \ldots, \ldots\}, \mathrm{r}=$ pseudorandom $(0,1)$.

(a) CW rotation, following slightly chaotic trajectories, slowly expanding.

(b) Interaction with the wall of the inner box reduces the chaos, producing more uniform trajectories.

(c) When the inner wall is removed, the system is completely stable.

(d) An instantaneous image of the individual vortices, rotating uniformly around their center as a rigid body. 
(a)
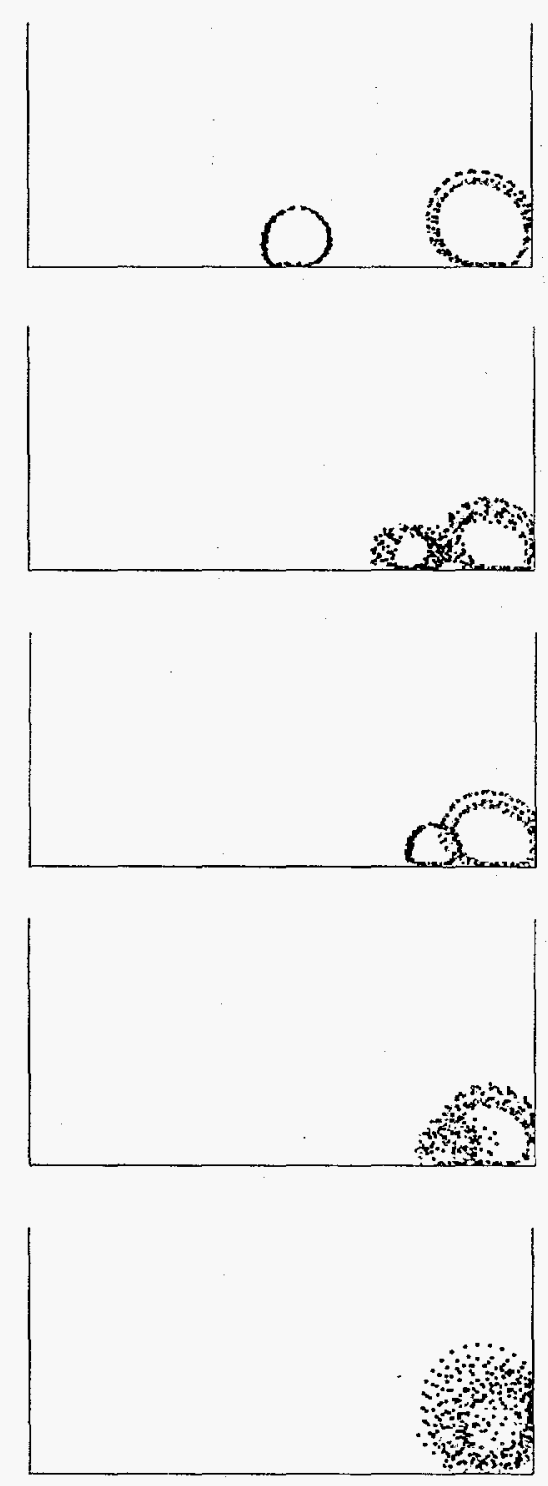

(b)
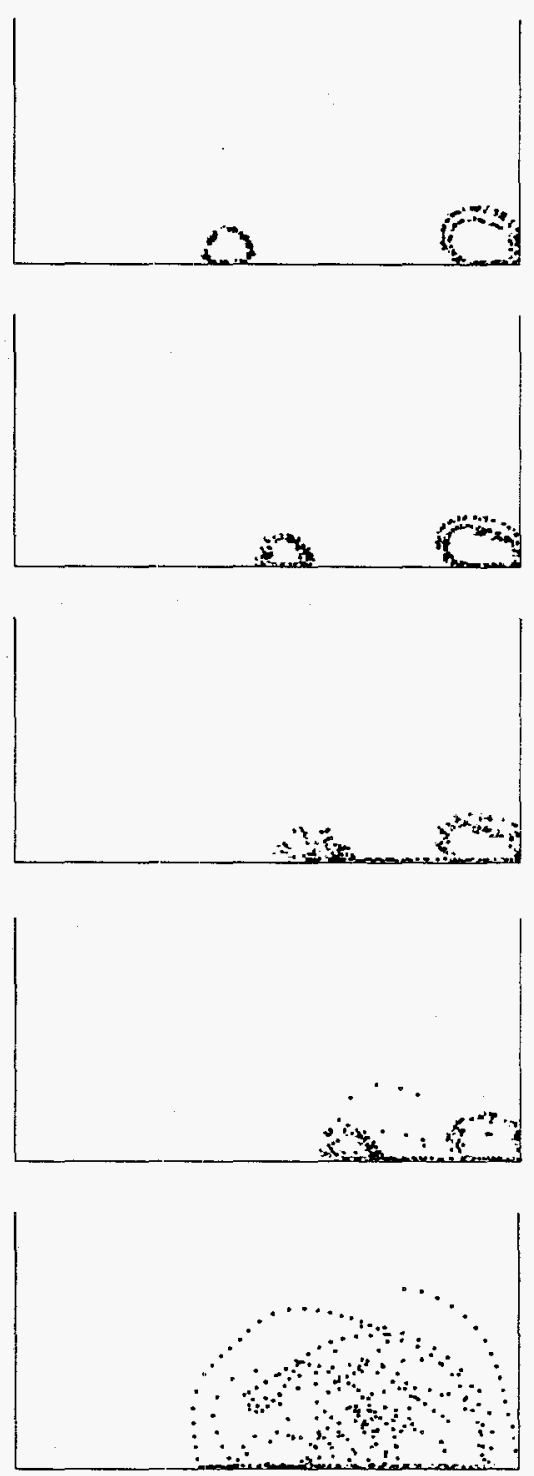

Figure 15 - Simulation of biological processes. The system is $\mathbf{P}=\{2 \mathrm{M}+2 \mathrm{~F}\}, \mathrm{S}=\{0, \mathrm{~s},-\mathrm{s}, 0\}$.

(a) $s=0.1$. Fusion

(b) $\mathrm{s}=0.7$. Filament-induced disintegration. 


\section{UNLIMITED RELEASE}

INITIAL DISTRIBUTION:

\begin{tabular}{|c|c|}
\hline MS0724 & D. L. Hartley, 6000 \\
\hline MS9001 & J. C. Crawford, 8000 \\
\hline MS9002 & P. N. Smith, 8500 \\
\hline MS9003 & D. L. Crawford, 8900 \\
\hline MS9004 & M. E. John, 8100 \\
\hline MS9005 & J. B. Wright, 5300 \\
\hline MS9006 & G. E. Ives, 5300 \\
\hline MS9037 & R. J. Detry, 8200 \\
\hline MS9054 & W. J. McLean, 8300 \\
\hline MS9103 & G. Thomas, 8111 \\
\hline MS9105 & L. A. Hiles, 8400 \\
\hline MS9108 & T. M. Dyer, 8800 \\
\hline MS9141 & S. Johnston, 8103 \\
\hline MS9163 & W. Bauer, 8302 \\
\hline MS9201 & L. D. Brandt, 8112 \\
\hline MS9201 & R. J. Gallagher, 8114 \\
\hline MS9214 & L. M. Napolitano, Jr., 8117 \\
\hline MS9214 & C. L. Bisson, 8117 \\
\hline MS9214 & M. E. Colvin, 8117 \\
\hline MS9214 & E. Friedman-Hill, 8117 \\
\hline MS9214 & R. S. Judson, 8117 \\
\hline MS9214 & W. P. Kegelmeyer, 8117 \\
\hline MS9214 & C. F. Melius, 8117 \\
\hline MS9214 & J. C. Meza, 8117 \\
\hline MS9214 & R. W. Schmieder, 8117 (10) \\
\hline MS9401 & R. C. Wayne, 8700 \\
\hline MS9403 & M. I. Baskes, 8712 \\
\hline MS9410 & J. C. Swearengen, 8113 \\
\hline MS9901 & L. A. West, 8600 \\
\hline MS9904 & W. D. Wilson, 8703 \\
\hline MS9021 & Technical Communications Department, 8535 for OSTI (10) \\
\hline MS9021 & $\begin{array}{l}\text { Technical Communications Department, 8535/Technical Library, } \\
\text { MS0899, } 13414\end{array}$ \\
\hline MS0899 & Technical Library, 13414 (4) \\
\hline MS9018 & Central Technical Files, 8523-2 (3) \\
\hline
\end{tabular}

